\title{
Revision of the convective transport module CVTRANS 2.4 in the EMAC atmospheric chemistry-climate model
}

\author{
H. G. Ouwersloot ${ }^{1}$, A. Pozzer ${ }^{1}$, B. Steil ${ }^{1}$, H. Tost ${ }^{2}$, and J. Lelieveld ${ }^{1}$ \\ ${ }^{1}$ Atmospheric Chemistry Department, Max Planck Institute for Chemistry, Mainz, Germany \\ ${ }^{2}$ Institute for Atmospheric Physics, University of Mainz, Mainz, Germany \\ Correspondence to: H. G. Ouwersloot (huug.ouwersloot@mpic.de)
}

Received: 9 March 2015 - Published in Geosci. Model Dev. Discuss.: 8 April 2015

Revised: 26 June 2015 - Accepted: 16 July 2015 - Published: 5 August 2015

\begin{abstract}
The convective transport module, CVTRANS, of the ECHAM/MESSy Atmospheric Chemistry (EMAC) model has been revised to better represent the physical flows and incorporate recent findings on the properties of the convective plumes. The modifications involve (i) applying intermediate time stepping based on a settable criterion, (ii) using an analytic expression to account for the intra-time-step mixing ratio evolution below cloud base, and (iii) implementing a novel expression for the mixing ratios of atmospheric compounds at the base of an updraft. Even when averaged over a year, the predicted mixing ratios of atmospheric compounds are affected considerably by the intermediate time stepping. For example, for an exponentially decaying atmospheric tracer with a lifetime of 1 day, the zonal averages can locally differ by more than a factor of 6 and the induced root mean square deviation from the original code is, weighted by the air mass, higher than $40 \%$ of the average mixing ratio. The other modifications result in smaller differences. However, since they do not require additional computational time, their application is also recommended.
\end{abstract}

\section{Introduction}

A key process in global modeling of atmospheric chemistry and climate is the vertical exchange of air (Lelieveld and Crutzen, 1994). Convective vertical motions redistribute energy, moisture and reactive trace species between different vertical layers within the troposphere. For clear sky conditions, this transport between e.g., Earth's surface and the top of the troposphere acts on timescales on the order of weeks. However, moist convective transport associated with cumuli- form clouds reduces it to time periods of hours (Lawrence and Rasch, 2005; Tost et al., 2010). Especially short-lived atmospheric compounds are strongly affected. Although important, the convective clouds cannot be explicitly resolved in general circulation models and need to be parameterized (e.g., Arakawa, 2004; Kim et al., 2012). Useful tools to derive and check these parameterizations are large-eddy simulation (LES) models that operate in smaller domains with a higher resolution (e.g., Bechtold et al., 1995; Siebesma and Cuijpers, 1995; Ouwersloot et al., 2013).

Here, we revise the parameterization in the convective transport module (CVTRANS; Tost et al., 2010) of the ECHAM/MESSy Atmospheric Chemistry (EMAC) model (Jöckel et al., 2006). This module is based on the bulk formulation for convective plumes introduced by Yanai et al. (1973) and treated and validated by Lawrence and Rasch (2005). While the original implementation already performs satisfactorily for weak to moderate convective transport, for strong convective transport the calculated mass transfer in one time step can exceed the total air mass of the plume at that location. When this happens, the updraft mass flux at an interface level is limited to transport exactly the total mass of the plume at the grid level below. This causes a misrepresentation of the actual physical flows and replenishes the air of entire grid cells in one time step, resulting in a too coarse calculation and unrealistic trace gas venting. By introducing intermediate time stepping in the module we remedy and quantify this issue. Additionally, an analytic expression is added to further account for intra-time-step changes of the air properties below the cloud base. Finally, it was found in a recent LES study (Ouwersloot et al., 2013) that cloudinduced large-scale atmospheric structures in the sub-cloud 
layer can affect the properties of the air that enters the convective plumes from below. The improvement to the convective transport parameterization proposed in this study is applied here as well. In addition to assessing the effects of the aforementioned revisions, we evaluate the impact of a different convective cloud cover representation on convective transport.

In Sect. 2 we describe the model and applied modifications. The setup to study the induced changes is presented in Sect. 3. These differences are then quantified and discussed in Sect. 4.

\section{Model}

\subsection{Original representation of convection}

In this study we apply and improve version 2.50 of the MESSy (Modular Earth Submodel System) framework (Jöckel et al., 2005, 2010), which is an interface structure that connects a base model to various submodels. Although our modifications are applicable to different base models as well, we validate the results using the EMAC model, first described by Jöckel et al. (2006). This system combines MESSy with version 5.3.02 of the European Centre Hamburg general circulation model (ECHAM5; Roeckner et al., 2006).

The moist convective transport for tracers other than water is calculated by the CVTRANS submodel (Tost et al., 2010), which represents the bulk formulation for convective plumes described by Lawrence and Rasch (2005). A single plume, also referred to as "leaky pipe", is considered for the updrafts and downdrafts separately. These plumes can laterally entrain and detrain at every level, resulting in a vertical mass flux that varies with height. The fluxes themselves, in $\mathrm{kg} \mathrm{m}^{-2} \mathrm{~s}^{-1}$, are not calculated in CVTRANS, but are gathered from the CONVECT submodel (Tost et al., 2006).

In the algorithm, the properties of the air that detrains from the plumes are determined according to ${ }^{1}$

$C_{\text {up , det. }}^{k}=\frac{\left(D_{\mathrm{up}}^{k}-f_{\mathrm{d}} E_{\mathrm{up}}^{k}\right) C_{\mathrm{up}}^{k+1}+f_{\mathrm{d}} E_{\mathrm{up}}^{k} C_{\mathrm{env} .}^{k}}{D_{\mathrm{up}}^{k}}$,
$C_{\text {down, det. }}^{k}=C_{\text {down }}^{k}$,

where $k$ is the height index, decreasing with altitude. The subscripts up, down and env. indicate properties of respectively the updraft, the downdraft and the ambient air in the cloud environment. If additionally the subscript det. is used, the variable represents the property of air that is detrained from the plume in that grid cell. $C$ is the mixing ratio (in mol mol ${ }^{-1}$ ), and $D$ and $E$ are respectively the rates of detrainment from and entrainment into the convective plume

\footnotetext{
${ }^{1}$ Note that (only) the mass fluxes and mixing ratios in the up- and downdraft plumes are specified at the top interface of the indexed grid cell.
}

(in $\mathrm{kg} \mathrm{m}^{-2} \mathrm{~s}^{-1}$ ). Part of the air that is entrained in the updraft is detrained again in the same grid cell (Lawrence and Rasch, 2005). The fraction of entrained air in a layer that is detrained again in the same layer is denoted by $f_{\mathrm{d}}$. Although this fraction is dependent on multiple factors, including grid resolution, it is generally set to a value of 0.5 . If necessary, $f_{\mathrm{d}}$ is adapted to ensure that the detrained mass flux that originates from the entrained air, $f_{\mathrm{d}} E_{\mathrm{up}}^{k}$, never exceeds the total detrained mass flux, $D_{\mathrm{up}}^{k}$, and that $f_{\mathrm{d}} E_{\mathrm{up}}^{k}$ is high enough so that the total amount of detrained air from the plume, $D_{\text {up }}^{k}$, does not exceed $F_{\text {up }}^{k+1}+f_{\mathrm{d}} E_{\text {up }}^{k} . F^{k}$ is the mass flux (in $\mathrm{kg} \mathrm{m}^{-2} \mathrm{~s}^{-1}$ ) at the top interface of grid level $k$.

The mixing ratios in the plumes, which are also needed for Eqs. (1) and (2), are instantaneously calculated as

$$
\begin{aligned}
& C_{\mathrm{up}}^{k}=\frac{F_{\mathrm{up}}^{k+1} C_{\mathrm{up}}^{k+1}-D_{\mathrm{up}}^{k} C_{\mathrm{up}, \text { det. }}^{k}+E_{\mathrm{up}}^{k} C_{\mathrm{env} .}^{k}}{F_{\mathrm{up}}^{k}} \\
& C_{\text {down }}^{k+1}=\frac{F_{\text {down }}^{k} C_{\text {down }}^{k}-D_{\text {down }}^{k} C_{\text {down, det. }}^{k}+E_{\text {down }}^{k} C_{\mathrm{env} .}^{k}}{F_{\text {down }}^{k+1}} .
\end{aligned}
$$

The mixing ratio in the updraft plume is initialized at the lowest level where the mass flux exceeds 0 , indicated by index $k_{\mathrm{b}}$. In the original CVTRANS 2.3 code

$$
C_{\mathrm{up}}^{k_{\mathrm{b}}}=C_{\mathrm{env}}^{k_{\mathrm{b}}} \text {. }
$$

The temporal evolution of the mixing ratios in the grid cells parts that are affected by the plumes is expressed by

$$
\begin{aligned}
& C_{\text {env. }}^{k}(t+\Delta t)=\frac{M_{\text {orig }}^{k}}{M^{k}} C_{\text {env. }}^{k}(t)+\frac{\Delta t}{M^{k}} \\
& \times\left(\left(F_{\text {up }}^{k}-F_{\text {down }}^{k}\right) C_{\text {env. }}^{k-1}+D_{\text {up }}^{k} C_{\text {up, det. }}^{k}+D_{\text {down }}^{k} C_{\text {down, det. }}^{k}\right)
\end{aligned}
$$

where $\Delta t$ is the time step and $M_{\text {orig }}$ is the mass per unit area of air (in $\mathrm{kg} \mathrm{m}^{-2}$ ) whose mixing ratio is not altered due to the plumes in one time step. This is calculated as

$$
M_{\text {orig }}^{k}=M^{k}-\Delta t\left(\left(F_{\text {up }}^{k}-F_{\text {down }}^{k}\right)+D_{\text {up }}^{k}+D_{\text {down }}^{k}\right) .
$$

$M$ without subscript is the total mass per unit area of air in which plumes occur in the grid cell, calculated as the total air mass per unit area in that grid cell times a certain cover. This cover can be selected as 1 or as the more representative convective cloud cover, calculated in the CONVECT module.

\subsection{Modifications to CVTRANS 2.4}

\subsubsection{Intermediate time steps}

If the vertical mass fluxes are very strong, $M_{\text {orig }}^{k}$ tends to 0 and the discretization does no longer suffice. Moreover, if $F_{\text {up }}^{k}$ exceeds $\frac{M^{k}}{\Delta t}$, it is truncated to that value in the CVTRANS 2.3 calculations to prevent instabilities and negative mixing ratios that may arise. However, as a result the physical 
flow is no longer properly represented. To remedy these issues we introduce intermediate time stepping in CVTRANS 2.4, where we divide the global time step in sub-time steps with length $\Delta t_{\text {sub. }}$. The amount of sub-time steps per global time step is determined per vertical column to ensure that at every level, $k$,

$F_{\text {up }}^{k} \Delta t_{\text {sub }}<f_{\text {maxfrac }} \min \left(M^{k}, M^{k-1}\right)$.

Here, $f_{\text {maxfrac }}$ is an a priori chosen fraction of $M$ that is allowed to leave the grid cell through the upward plume per sub-time step. This fraction is set in the updated CVTRANS namelist. For every horizontal location the convective transport in the column is calculated independently in CVTRANS 2.4 using the locally required amount of sub-steps.

\subsubsection{Analytic expression at cloud base}

Near the convective cloud base, we can account for recirculation effects within a single time step in a computationally less inexpensive manner by applying an analytic solution for the sub-cloud mixing ratio evolution. At cloud base level $k_{\mathrm{b}}$, $C_{\text {env }}^{k_{\mathrm{b}}}$ evolves in time according to

$$
\frac{\partial}{\partial t} M^{k_{\mathrm{b}}} C_{\mathrm{env}}^{k_{\mathrm{b}}}=\underbrace{-F_{\mathrm{up}}^{k_{\mathrm{b}}} C_{\mathrm{env}}^{k_{\mathrm{b}}}}_{\text {upward plume }}+\underbrace{F_{\mathrm{up}}^{k_{\mathrm{b}}} C_{\mathrm{env}}^{k_{\mathrm{b}}-1}}_{\text {compensating subsidence }},
$$

since air leaves the grid cell with properties of the environmental air and is replenished by compensating subsidence with properties of the environmental air in the overlying grid cell. During the time step the mass and mass fluxes do not change, resulting in

$$
\begin{aligned}
& \left\langle C_{\mathrm{env}}^{k_{\mathrm{b}}}\right\rangle=C_{\mathrm{env}, 0}^{k_{\mathrm{b}}-1}+\left(C_{\mathrm{env}, 0}^{k_{\mathrm{b}}}-C_{\mathrm{env}, 0}^{k_{\mathrm{b}}-1}\right) \frac{1-\mathrm{e}^{-f_{\text {frac }}}}{f_{\text {frac }}}, \\
& f_{\text {frac }}=\frac{F_{\mathrm{up}}^{k_{\mathrm{b}}} \Delta t_{\mathrm{sub}}}{M^{k_{\mathrm{b}}}}
\end{aligned}
$$

where \langle\rangle indicates a temporal average over the sub-time step and subscript 0 refers to the value at the start of the sub-time step. Using $\left\langle C_{\mathrm{env}}^{k_{\mathrm{b}}}\right\rangle$ instead of $C_{\mathrm{env}}^{k_{\mathrm{b}}}$ in Eq. (5) does not yield substantially different results if $\frac{F_{\mathrm{up}}^{k_{\mathrm{b}}} \Delta t_{\text {sub }}}{M^{k_{\mathrm{b}}}} \ll 1$. Otherwise, this revised representation accounts for the major influence of the updraft plume on the sub-plume mixing ratio evolution within the time step and for the resulting reduced impact of vertical mixing ratio gradients around the plume base.

\subsubsection{Altered concentrations at updraft base}

As a third modification, we include a recently published parameterization for the vertical transport of chemical reactants at the convective cloud base (Ouwersloot et al., 2013). Related to induced large-scale circulations in the convective boundary layer below the convective plumes, it was found that the mixing ratios of atmospheric chemical species at the base of the updraft plume, $C_{\mathrm{up}}^{k_{\mathrm{b}}}$, differ even more from $C_{\mathrm{env}}^{k_{\mathrm{b}}-1}$ than $C_{\mathrm{env}}^{k_{\mathrm{b}}}$. Considering $C_{\mathrm{env}}^{k_{\mathrm{b}}}$ to be representative for the mixing ratio in the sub-cloud layer, their Eq. (13) is applied by replacing our Eq. (5) by

$C_{\mathrm{up}}^{k_{\mathrm{b}}}=C_{\mathrm{env}}^{k_{\mathrm{b}}}+\left(f_{\mathrm{trans}}-1\right)\left(C_{\mathrm{env}}^{k_{\mathrm{b}}}-C_{\mathrm{env}}^{k_{\mathrm{b}}-1}\right)$,

where $f_{\text {trans }}$ is a namelist setting with a standard value of 1.23 (Ouwersloot et al., 2013). When both this parameterization and the analytic solution below the cloud base are applied, Eq. (5) is again replaced by Eq. (10), while Eq. (11) is updated to

$f_{\text {frac }}=\frac{f_{\text {trans }} F_{\mathrm{up}}^{k_{\mathrm{b}}} \Delta t_{\mathrm{sub}}}{M^{k_{\mathrm{b}}}}$.

These updated mixing ratios are only applied if the updraft plume is affected by convective boundary-layer dynamics. This is considered to be the case if the bottom of the plume is located below the boundary-layer height that is diagnosed by the TROPOP module or below a height limit that can be set in the CVTRANS namelist. In this study it is kept to the standard setting of $2500 \mathrm{~m}$.

\section{Simulation setup}

We performed numerical simulations with EMAC to quantify the impact of the various code modifications. In these simulations, the MESSy submodels that are listed in Table 1 have been enabled. Unless specified differently, standard settings are used. For illustration purposes, the convective transport is tested for the standard convection parameterization in EMAC, which is based on Tiedtke (1989) and Nordeng (1994). The simulations are all performed at the T63 horizontal resolution $(192 \times 96$ grid $)$ with 31 vertical hybrid pressure levels and a time step of $12 \mathrm{~min}$. The simulation period spans the years 2000 and 2001, of which the former year is considered spinup time. The initial state is prescribed by ECMWF (European Centre for Medium-Range Weather Forecasts) operational analysis data. To check the undisturbed effects of the applied modifications, no nudging is applied to meteorological data during the simulation.

Convective transport is evaluated using passive tracers with exponential decay and a constant spatially uniform emission pattern. The lifetimes of these tracers, $\tau$, are $1000 \mathrm{~s}$, 1 and $6 \mathrm{~h}$, and 1,2, 25 and 50 days and were chosen to represent various atmospheric compounds that are affected by convective transport. By prescribing passive, exponentially decaying tracers we prevent feedbacks between chemical species and meteorology and can focus on the relation between the modified convective transport and the lifetime of the tracers. Since processes in EMAC are mass conserving and these tracers are not chemically active, the total mass of a tracer at a given time is the same for each numerical experiment. 
Table 1. Optional MESSy submodels that are enabled for the numerical experiments.

\begin{tabular}{lll}
\hline Submodel & Executed process & Reference \\
\hline CLOUD & Original ECHAM5 cloud formation & Roeckner et al. (2006) \\
CONVECT & Convection & Tost et al. (2006) \\
CVTRANS & Convective tracer transport & Tost et al. (2010) and text \\
OFFEMIS & Prescribed emissions of trace gases & Kerkweg et al. (2006) \\
PTRAC & Prognostic tracers & Jöckel et al. (2008) \\
TNUDGE & Pseudo-emissions of tracers & Kerkweg et al. (2006) \\
TREXP & Exponentially decaying tracers & Jöckel et al. (2010) \\
TROPOP & Tropopause and boundary-layer diagnostics & Jöckel et al. (2006) \\
VISO & Diagnostics at isosurfaces & Jöckel et al. (2010) \\
\hline
\end{tabular}

Table 2. Description of the different numerical experiments. Listed are the differences in settings between the simulations and required computational time (in CPU hours). If $f_{\text {maxfrac }}$ is set to -, intermediate time steps are not enabled. The columns Analytic and Updraft denote respectively whether the analytic expression and the updated concentrations at the updraft base are applied. The applied cloud cover is either diagnosed in the CONVECT module or set to 1.

\begin{tabular}{lrlllr}
\hline Name & $f_{\text {maxfrac }}$ & Analytic & Updraft & Cloud cover & Time (CPU h) \\
\hline ORG & - & No & No & Diagnosed & 349 \\
I100 & 1.00 & No & No & Diagnosed & 386 \\
I050 & 0.50 & No & No & Diagnosed & 416 \\
I025 & 0.25 & No & No & Diagnosed & 514 \\
I015 & 0.15 & No & No & Diagnosed & 608 \\
I010 & 0.10 & No & No & Diagnosed & 748 \\
I005 & 0.05 & No & No & Diagnosed & 1175 \\
I001 & 0.01 & No & No & Diagnosed & 4544 \\
\hline ORGA & - & Yes & No & Diagnosed & 349 \\
I100A & 1.00 & Yes & No & Diagnosed & 383 \\
I050A & 0.50 & Yes & No & Diagnosed & 421 \\
I010A & 0.10 & Yes & No & Diagnosed & 763 \\
I001A & 0.01 & Yes & No & Diagnosed & 4864 \\
\hline UPDP & 0.50 & Yes & Yes & Diagnosed & 420 \\
UPDP & 0.01 & No & Yes & Diagnosed & 4360 \\
CC & 0.50 & Yes & No & 1 & 339 \\
CC & 0.01 & No & No & 1 & 435 \\
\hline
\end{tabular}

Multiple numerical experiments have been performed. Experiments whose name start with "ORG" do not use the intermediate time stepping, but if an experiment name starts with an "I", it does employ the intermediate time stepping and it is followed by a three-digit number that is equal to $100 \times f_{\text {maxfrac }}$. The most precise experiment, I001, thus sets $f_{\text {maxfrac }}$ to 0.01 . Note that in our analyses, $I 001$ is considered to represent convective tracer transport best and is used as the reference simulation to quantify deviations. If the numerical experiment is followed by an "A", the analytic expression for the temporal evolution of mixing ratios below the convective cloud base is applied as well. In general, the adapted convective transport near cloud base is not applied and we use the convective cloud cover as calculated in CONVECT to determine the fractions of the grid cells that are affected by the updraft and downdraft plumes. However, numerical experi- ments UPDP and CC, both based on numerical experiment I050A, are exceptions to this. In UPDP the adapted convective transport parameterization at the updraft plume base is enabled. In CC the convective transport is calculated using a convective cloud cover of 1 , representing the extreme case where convective plumes span entire grid cells. Note that the resulting mass transport per affected unit area is weaker and therefore applying intermediate time steps has less impact. To complete the quantification of differences, additional numerical experiments $\mathrm{UPDP}^{+}$and $\mathrm{CC}^{+}$are conducted, which are similar to UPDP and CC but based on experiment I001 instead of I050A. An overview of the different numerical experiments is presented in Table 2 .

While evaluating induced differences, only data averaged over 2001 is considered. Hence, we do not consider shortterm fluctuations but rather focus on long-term shifts re- 
Table 3. Weighted root mean square deviations between two numerical experiments. Results, expressed as percentages of the respective air-mass-weighted mixing ratios, are listed for the seven tracers.

\begin{tabular}{|c|c|c|c|c|c|c|c|c|}
\hline \multicolumn{2}{|c|}{ Comparison } & \multicolumn{7}{|c|}{ RMSD $(\%)$ for tracers with a lifetime of } \\
\hline Exp. 1 & Exp. 2 & $1000 \mathrm{~s}$ & $1 \mathrm{~h}$ & $6 \mathrm{~h}$ & 1 day & 2 days & 25 days & 50 days \\
\hline ORG & ORGA & 0.108 & 0.087 & 0.079 & 0.104 & 0.174 & 0.198 & 0.130 \\
\hline ORG & I001 & 7.462 & 11.022 & 28.156 & 41.170 & 39.442 & 10.536 & 6.145 \\
\hline ORG & $\mathrm{I} 100$ & 8.068 & 11.859 & 29.945 & 43.354 & 41.342 & 11.006 & 6.431 \\
\hline I001 & ORGA & 7.543 & 11.080 & 28.203 & 41.206 & 39.467 & 10.566 & 6.170 \\
\hline I001 & I001A & 0.005 & 0.005 & 0.004 & 0.003 & 0.002 & 0.001 & 0.001 \\
\hline I001 & I005 & 0.026 & 0.038 & 0.084 & 0.101 & 0.088 & 0.022 & 0.013 \\
\hline I001 & I010A & 0.028 & 0.057 & 0.161 & 0.208 & 0.183 & 0.044 & 0.027 \\
\hline I001 & I010 & 0.059 & 0.085 & 0.188 & 0.227 & 0.197 & 0.050 & 0.030 \\
\hline I001 & I015 & 0.092 & 0.133 & 0.291 & 0.351 & 0.306 & 0.077 & 0.047 \\
\hline I001 & I025 & 0.158 & 0.227 & 0.498 & 0.599 & 0.520 & 0.131 & 0.079 \\
\hline I001 & I050A & 0.160 & 0.326 & 0.883 & 1.119 & 0.982 & 0.237 & 0.142 \\
\hline I001 & I050 & 0.325 & 0.468 & 1.013 & 1.210 & 1.050 & 0.263 & 0.159 \\
\hline I001 & $\mathrm{I} 100 \mathrm{~A}$ & 0.339 & 0.668 & 1.725 & 2.142 & 1.872 & 0.453 & 0.273 \\
\hline I001 & I100 & 0.652 & 0.936 & 1.973 & 2.318 & 2.004 & 0.505 & 0.308 \\
\hline I050A & UPDP & 0.583 & 0.523 & 0.378 & 0.246 & 0.174 & 0.029 & 0.016 \\
\hline I001 & $\mathrm{UPDP}^{+}$ & 0.581 & 0.522 & 0.379 & 0.249 & 0.177 & 0.029 & 0.016 \\
\hline I050A & $\mathrm{CC}$ & 9.085 & 14.322 & 27.233 & 26.891 & 23.022 & 7.091 & 4.222 \\
\hline I001 & $\mathrm{CC}^{+}$ & 8.890 & 13.891 & 26.861 & 26.894 & 23.084 & 7.111 & 4.238 \\
\hline
\end{tabular}

lated to the different convective transport representations. For quantification, the root mean square deviation (RMSD) over the numerical grid is used, weighted by the air mass, $M$, in each grid cell. For two different simulations, denoted by indicators $\mathrm{A}$ and $\mathrm{B}$, the RMSD of a mixing ratio, $c$, is defined as

$$
\operatorname{RMSD}_{\mathrm{A}, \mathrm{B}}(c)=\sqrt{\frac{\sum_{i} \overline{M_{i}}\left(\overline{\mathrm{c}_{\mathrm{A}, i}}-\overline{c_{\mathrm{B}, i}}\right)^{2}}{\sum_{i} \overline{M_{i}}}},
$$

where indicator $i$ iterates over the individual grid cells and an overbar denotes a temporal average over 2001. To put into perspective, the RMSD is always expressed as a percentage of the air-mass-weighted mixing ratio, $\sum_{i}\left(\overline{M_{i}} c_{i}\right) / \sum_{i} \overline{M_{i}}$. Note that the air-mass-weighted mixing ratio is the same for all numerical experiments since we evaluate chemically inert species with constant emissions.

\section{Results}

In Sect. 4.1 the effect of intermediate time steps on the atmospheric compounds is shown. The effect of using the analytic expression, for the temporal mean mixing ratio during a time step below the updraft plume, is discussed in Sect. 4.2. Subsequently, the optimal settings for intermediate time steps and the analytic expression are determined in Sect. 4.3 for the current numerical setup. The changes induced by considering the updated parameterization for mixing ratios at the updraft plume base and a different convective cloud cover are treated in Sects. 4.4 and 4.5, respectively.

The weighted root mean square deviations between different numerical experiments are listed in Table 3.

\subsection{Intermediate time steps}

As can be seen from Table 3, the strongest deviations are found for a lifetime of 1 or 2 days. This is related to the timescale of convective transport being on the same order of magnitude. Atmospheric compounds with longer lifetimes are generally well mixed with height and their distribution is therefore less affected by convective transport. Shorter-lived species are mainly concentrated near the sources at Earth's surface, resulting in low mixing ratios and, consequently, low absolute deviations where convective transport is active. However, even for short ( $\tau=1000 \mathrm{~s})$ or long ( $\tau=50$ days $)$ lifetimes, the root mean square deviations of the $2001 \mathrm{av}$ eraged mixing ratio are over $5 \%$ of the respective weighted mean mixing ratios.

In Fig. 1, the 2001 averaged mixing ratio for the atmospheric compound with a lifetime of 1 day is depicted at the $700 \mathrm{hPa}$ level. This level is generally located in the lower free troposphere, above the sub-cloud layer or clear-sky atmospheric boundary layer, except for areas at high elevation where the surface pressure is low. Since the atmospheric compound is emitted at Earth's surface and decays much faster than the timescale of vertical exchange for clear sky conditions, its mixing ratio is low in the free troposphere 

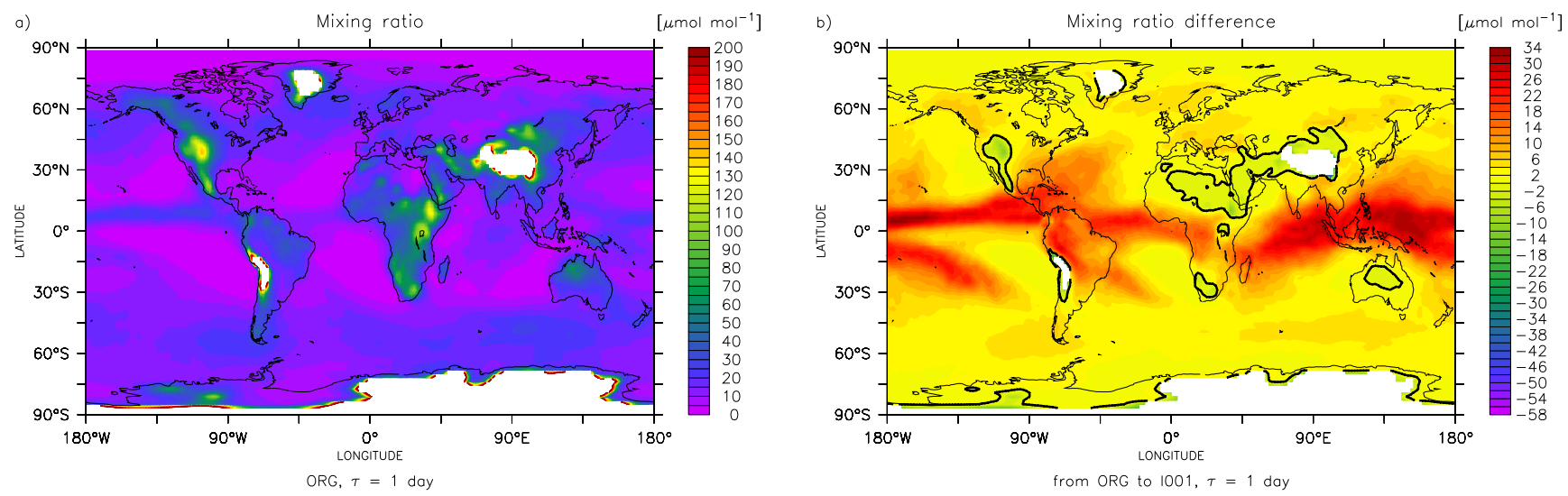

Figure 1. Horizontal distribution of the decaying scalar with a lifetime of 1 day, averaged over 2001 at $700 \mathrm{hPa}$. Shown are (a) the distribution for the ORG numerical experiment and (b) the mixing ratio difference for I001 compared to ORG.

compared to the atmospheric boundary layer, except for locations where convective transport is active. From Fig. 1a it can be seen that indeed relatively high mixing ratios are found in regions that are either characterized by a high elevation, thus evaluating boundary-layer air, or by more active convection, such as the intertropical convergence zone, the South Pacific convergence zone and the westerly storm tracks.

In the ORG numerical experiment, convective transport is capped when the upward mass flux transports more air in one time step than is present in the underlying grid cell. This nonphysical capping of the flow can be removed when intermediate time steps are enabled. As shown in Fig. 1b, this results in enhanced vertical transport and thus higher free tropospheric mixing ratios, particularly in the areas with strong convection. Supporting images are presented in Fig. 1 of the Supplement. In the boundary layer, as illustrated by the areas with high elevation, the mixing ratios become slightly lower due to the enhanced vertical transport. The increase in mixing ratios in the free troposphere are of the same order as the mixing ratios in the ORG numerical experiment and the final mixing ratios in I001 can be up to a factor of 5 higher (not shown). This high factor is mainly due to the low mixing ratios in ORG at those locations, which yields large relative differences for small absolute mixing ratio differences. Therefore, the air-mass-weighted root mean square deviation of the 2001 averaged mixing ratios is used for the quantification, which is equal to $43 \%$ of the air-mass-weighted mixing ratio for the tracer with a lifetime of 1 day.

The substantial change in the representation of convective transport with intermediate time steps is also clear from Fig. 2, with changes over $500 \%$ in the yearly and zonally averaged mixing ratios compared to the ORG numerical experiment. Although these high, relative differences typically occur in regions with relatively low mixing ratios, they can be compared to similar figures for the effects of different convection parameterizations (e.g., Fig. 2 in Tost et al., 2010) and of using an ensemble plume model instead of a bulk plume model (e.g., Fig. 4 in Lawrence and Rasch, 2005). Even though mixing ratios were averaged over shorter periods in those studies, much lower relative changes were found with maximum differences between 20 and $100 \%$. That the consequential variations in representing convective transport applied by Lawrence and Rasch (2005) and Tost et al. (2010) yield smaller differences in the distributions of trace species emphasizes the importance of applying the intermediate time steps.

Note from Table 3 that coarser intermediate time steps, e.g., I100, yield similar differences, compared to ORG as I001, and that the deviations between I001 and I100 are more than 10 times smaller. This shows that the strongest effect results from the convective transport by the updraft plume no longer being capped, since in I100 entire grid cells can still be depleted of air in individual sub-time steps. Since within each intermediate time step I100 does not account for the recirculation of air and the mass of the entire grid cell can be removed, the effectiveness of convective transport is actually overestimated, while it was underestimated in ORG. This is why the RMSD values between I100 and ORG are slightly higher than those between I001 and ORG. To better account for this recirculation, lower values for $f_{\text {maxfrac }}$ can be chosen and the analytic expression for the temporal mean mixing ratio below the convective cloud base can be employed.

\subsection{Analytic expression}

By applying the analytic expression for the (sub-)time step average mixing ratio below the cloud base of Eq. (10), we can account for the subsiding motions that compensate for mass loss below the cloud base due to the updraft plumes within this (sub-)time step. Through this process, air is replenished and the mixing ratio at the updraft plume base is not only determined by the environmental mixing ratio below plume base but also by the environmental mixing ratio in the first layer aloft. This effect is stronger with higher updraft mass 

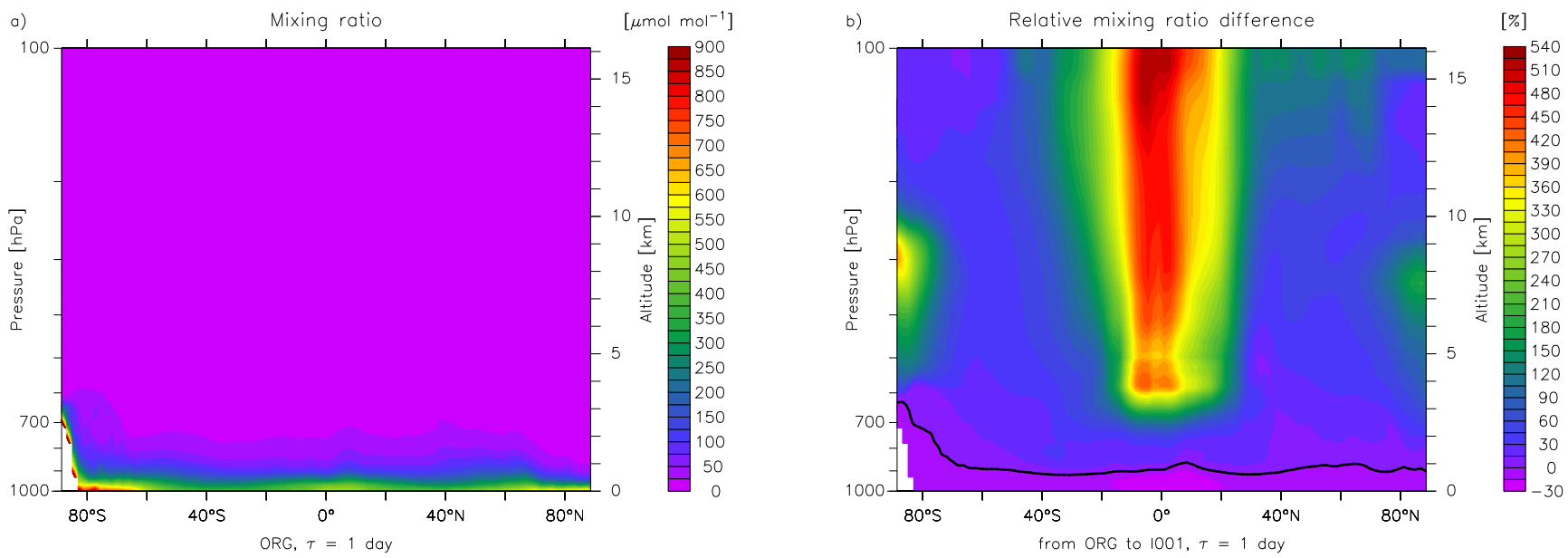

Figure 2. Decaying scalar with a lifetime of 1 day, averaged zonally and over 2001. Shown are (a) the distribution for the ORG numerical experiment and (b) the relative mixing ratio difference for I001 compared to ORG.

fluxes. As a result, it will no longer occur that the entire air mass in the grid cell below the plume base is replaced by environmental air from the grid cell above the plume base.

Since part of the air at the updraft plume base now originates from the environment above cloud base, the effect of vertical mixing by convective transport is reduced. This results in stronger vertical gradients with higher mixing ratios near the surface and higher mixing ratios in the upper troposphere, as confirmed in Fig. 3. Because vertical transport is underestimated in ORG, due to the capping of the mass fluxes of the updraft plumes, the RMSD between ORGA and I001 is actually higher than between ORG and I001. However, for all numerical experiments with intermediate time stepping, where mass fluxes are not capped, the RMSD compared to I001 decreases when the analytic expression is employed. This effect is especially influential for shorter lived species, roughly halving the RMSD compared to the reference case for $\tau=1000 \mathrm{~s}$.

As most clearly illustrated by the RMSD between ORG and ORGA in Table 3, the analytic expression increases in significance when the lifetime of the tracer is shorter. We hypothesize that this is related to the vertical distribution of the exponentially decaying tracers. For shorter lifetimes, a greater part of these tracers is located in the lower troposphere, where the effect of the represented recirculation around the cloud base is strongest.

\subsection{Performance}

While the dynamics are best represented by using intermediate time stepping with a low $f_{\text {maxfrac }}$ in combination with the analytic expression of Eq. (10), these settings can be computationally expensive. Therefore, an optimal setting should be chosen that limits the amount of required computational time, but results in low RMSD values compared to the reference simulation, I001. For illustration, these values are

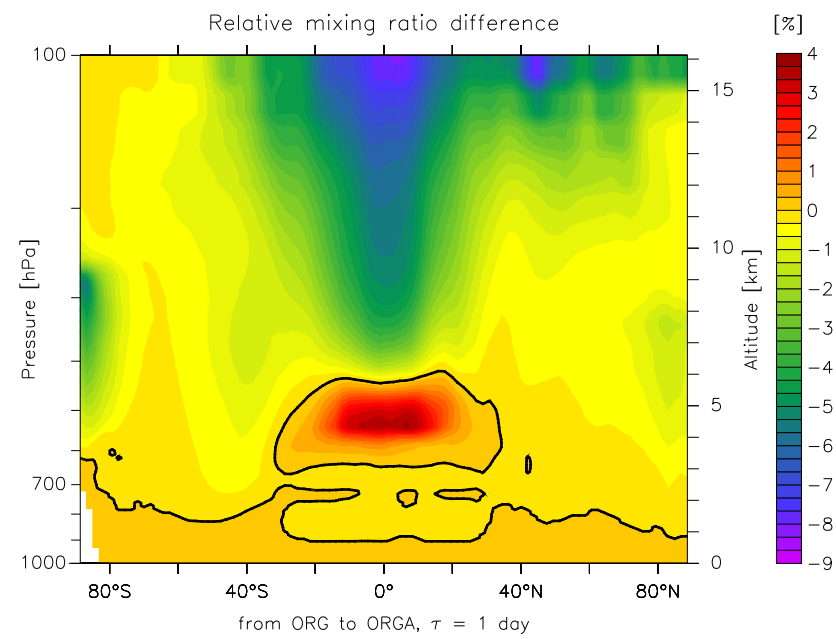

Figure 3. Relative difference in zonally and 2001 averaged mixing ratios for ORGA compared to ORG. Results are shown for the tracer with a lifetime of 1 day.

shown as a function of computational time in Fig. 4 for the tracers with lifetimes of $1000 \mathrm{~s}$ and 1 day. For this we take the computational time that each respective numerical experiment needed to finish the 2 year simulation with the settings listed in Sect. 3.

The RMSD is roughly proportional to the value of $f_{\text {maxfrac }}$, while the extra required computational time with respect to ORG scales inversely to $f_{\text {maxfrac }}$. In this setup we select $f_{\text {maxfrac }}=0.50$ as most desirable for further analyses, since the error is halved compared to I100 with only a limited increase in computational time. When other computationally expensive modules (e.g., chemical reactions) are enabled, the increase in computational expense for the CVTRANS mod- 
a)

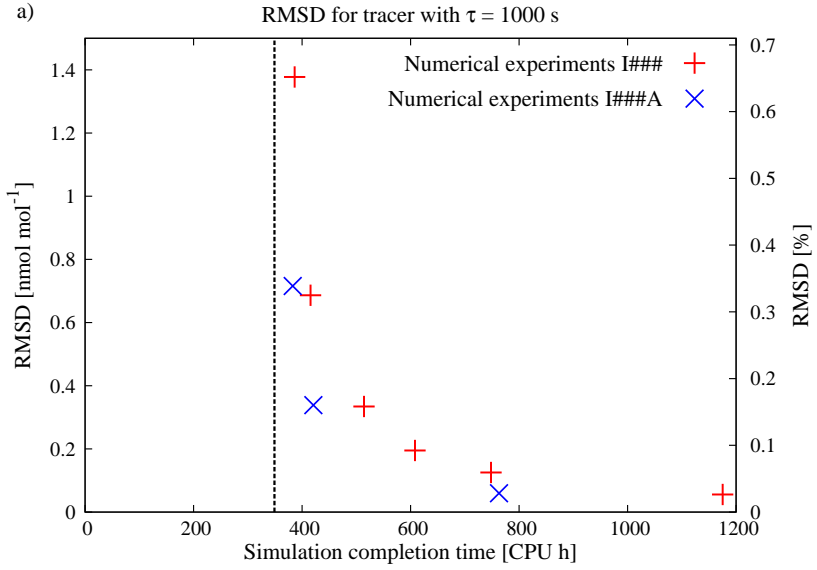

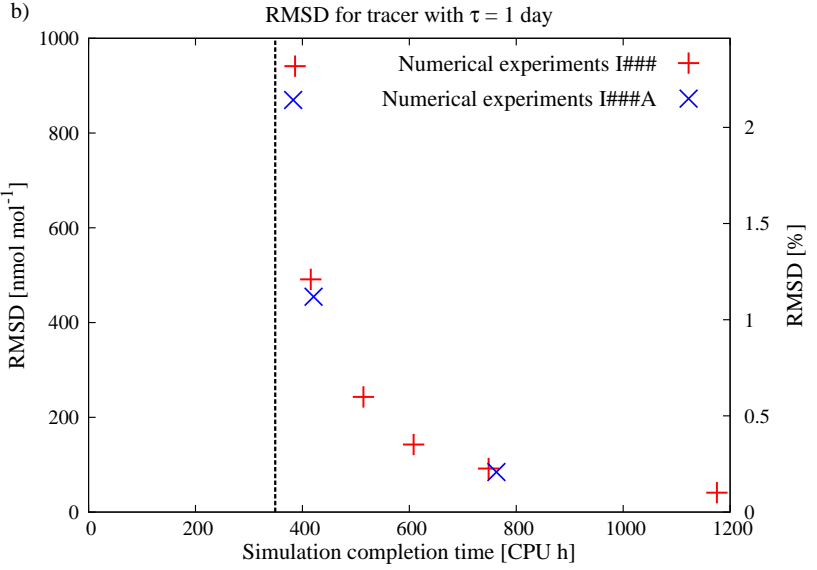

Figure 4. Root mean square deviations of the 2001 averaged mixing ratios compared to reference case I001 for decaying scalars with a lifetime of (a) $1000 \mathrm{~s}$ and (b) 1 day. On the vertical axes, the RMSD is expressed in both absolute numbers and as percentages of the airmass-weighted mixing ratios. On the horizontal axis, the computational time used by the numerical experiments is depicted. The red pluses, from left to right, represent the numerical experiments I100, I050, I025, I015, I010 and I005. The blue crosses represent the numerical experiments I100A, I050A and I010A. The dotted line expresses the computational time used by ORG.

ule becomes even less consequential for the total simulation completion time and lower $f_{\text {maxfrac }}$ values can be chosen.

Applying the analytic expression does not change the computational time substantially but always improves the results when intermediate time stepping is applied. This improvement reduces the RMSD only by a small amount $(\sim 10 \%)$ for longer-lived tracers but rather considerably for shorterlived species (e.g., $\sim 50 \%$ for $\tau=1000 \mathrm{~s}$ ).

As we find that setting $f_{\text {maxfrac }}$ to 0.50 and applying the analytic expression results in the optimal tradeoff between required computational time and resulting RMSD, I050A will be used as base numerical experiment and reference to study the effects of the adapted mixing ratio parameterization at the base of the updraft plume (Sect. 4.4) and of using a different convective cloud cover (Sect. 4.5).

\subsection{Adapted updraft plume base}

Here we apply the improved representation for mixing ratios in the base of the updraft plume that was presented by Ouwersloot et al. (2013). In Fig. 5, the resulting deviations in zonally and yearly averaged mixing ratios are shown for atmospheric tracers with lifetimes of $1000 \mathrm{~s}$ and 1 day. In general, stronger relative deviations in these mixing ratios are found for the tracers with a lower atmospheric lifetime. However, the strongest of these relative differences are located in areas with low mixing ratios, so that their impact on the total root mean square deviation is low. Although the strongest impact on this metric is also found for tracers with the lowest lifetime, for all atmospheric tracers the RMSD is less than $0.6 \%$ of the air-mass-weighted mixing ratio. The reason that faster decaying tracers are affected more strongly is the same as for applying the analytic expression for (sub-)time step average mixing ratios below the cloud base (Sect. 4.2). Both processes affect the efficiency of convective transport near the base of the updraft plume.

The low deviations are most likely related to the limited vertical mixing ratio gradients around the cloud base. Except for a $\tau$ of $1000 \mathrm{~s}$ or $1 \mathrm{~h}$, the RMSD related to applying the improved representation at the updraft plume base is always less than the RMSD between the most accurate numerical experiment, I001, and the selected base numerical experiment for the intercomparison, I050A. Also, for these shorter lifetimes the RMSD values between I050A and UPDP are lower than the effect of using very coarse intermediate time steps, quantified by the RMSD between I001 and I100. From this perspective the improvement is not very important. However, this small improvement comes without enhanced computational cost. Furthermore, this metric was evaluated globally using data that was averaged over 2001. Local, instantaneous differences can be more noteworthy, e.g., on the order of $10 \%$ in the lowest kilometer of the atmosphere. Therefore, we still recommend to apply this updated calculation.

\subsection{Convective cloud cover}

As indicated in Sect. 3, in the previously treated numerical experiments the convective transport is concentrated in a fraction of the grid cell, determined by the convective cloud cover. The current calculation of convective cloud cover in EMAC is rudimentary; assuming that

$c_{\text {conv }}^{k}=\frac{F_{\text {up }}^{k}}{\rho_{\text {air }}^{k} v_{\text {upd }}}$,

where $c_{\text {conv }}$ is the convective cloud cover, $\rho_{\text {air }}$ is the density of air (in $\mathrm{kg} \mathrm{m}^{-3}$ ), and $v_{\text {upd }}$ is the updraft velocity that is assumed to be constant at $1 \mathrm{~ms}^{-1}$. Alternatively, in CVTRANS the convective transport can be distributed over the 

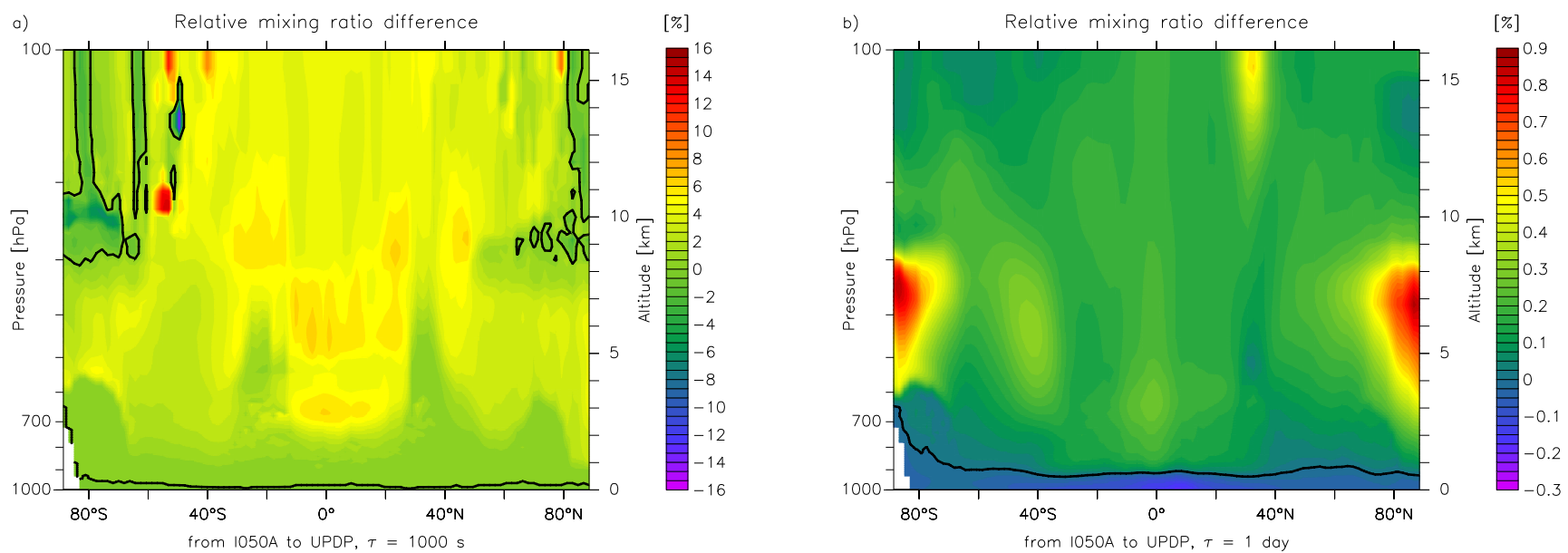

Figure 5. Relative difference in zonally and 2001 averaged mixing ratios for UPDP compared to I050A. Results are shown for the tracers with lifetimes of (a) $1000 \mathrm{~s}$ and (b) 1 day.
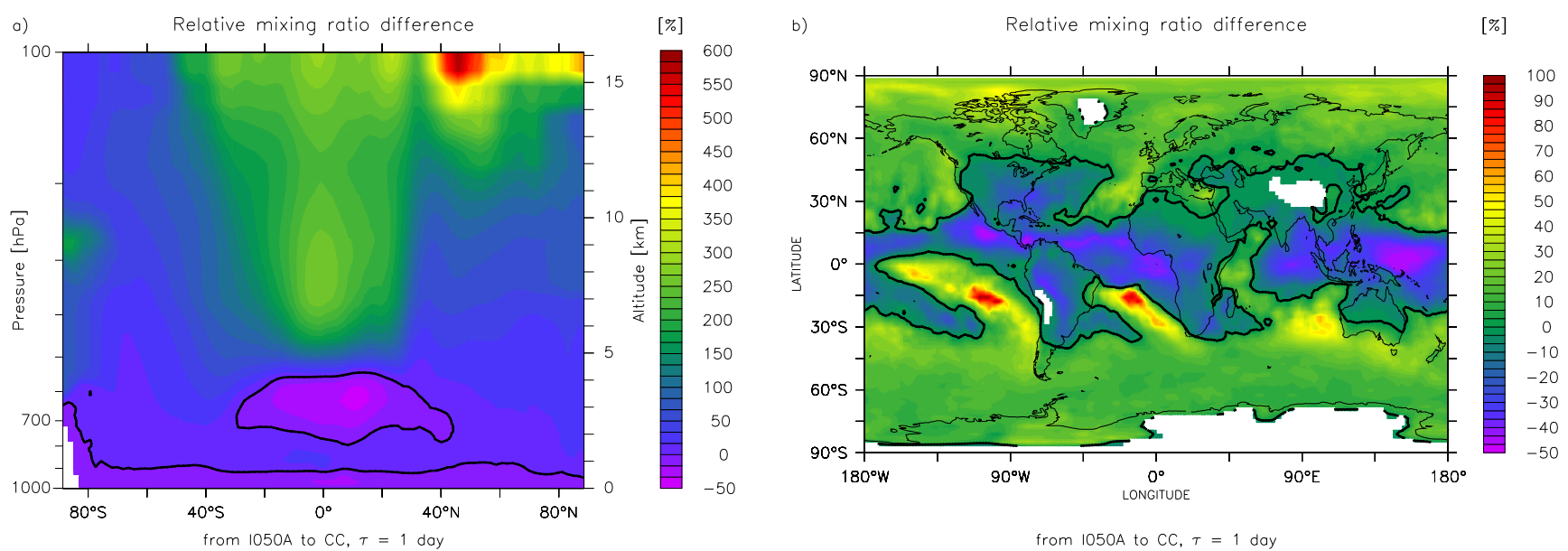

Figure 6. Relative difference in the 2001 averaged mixing ratio of the atmospheric tracer with a lifetime of 1 day for CC compared to I050A. Results are shown for (a) the zonally averaged data and (b) the difference at the $700 \mathrm{hPa}$ level.

entire grid cell, which is identical to assuming a convective cloud cover of 1 . Considering that both settings are possible and that the current calculation of convective cloud cover could be updated, it is worth investigating the impact of this chosen convective cloud cover. To investigate this, numerical experiment CC is performed, which is identical to I050A except for distributing the convective transport over the entire grid cell.

Due to the larger area, the plumes transport a smaller fraction of the affected air mass and there are less recirculation effects. Therefore, the vertical transport from the lower cloud layers to the upper cloud layers becomes more effective and especially higher mixing ratios are found in the upper troposphere, as shown in Fig. 6a. In areas of strong convection, this leads to decreased mixing ratios in the lower altitude regions where convective transport is active. This effect is visible from the averaged mixing ratios at a pressure of $700 \mathrm{hPa}$ in
Fig. 6b. Supporting images are presented in Fig. 2 of the Supplement. Similar to applying intermediate time stepping, the strongest effects are found for atmospheric tracers with intermediate lifetimes. The reasons are similar, since the transport is affected in the entire plume and the effective vertical transport is enhanced. The shift in the tracer lifetime that corresponds with the most pronounced change, towards a $\tau$ of between $6 \mathrm{~h}$ and 1 day, is caused by the strongly affected lower part of the convective plumes. For this assumed convective cloud cover of 1 , enabling intermediate time steps yields smaller differences (RMSD $<1 \%$ ) due to the weaker local mass transport.

In total, the effect of using a different convective cloud cover definition is substantial, with RMSD values ranging from 4 (for $\tau=50$ days) to $27 \%$ of the air-mass-weighted mixing ratio. This shows that it is important to apply a valid 
representation of the convective cloud cover when evaluating convective transport.

\section{Conclusions and outlook}

We presented various modifications to the CVTRANS module in the EMAC model to update and revise the representation of convective transport of atmospheric compounds. The new, optional functionality consists of (i) intermediate time stepping when updraft mass fluxes are too strong compared to the air mass in individual grid cells, (ii) an analytic expression that accounts for the intra-(sub-)time-step evolution of air properties below the base of the convective plume, and (iii) a recently published parameterization for the mixing ratios of atmospheric compounds at the updraft base.

It was demonstrated that applying the intermediate time stepping results in a substantial difference in atmospheric mixing ratios, even when averaged over 2001. The most important effect turned out to be that physical flows no longer need to be capped due to numerical limits. For high values of $f_{\text {maxfrac }}$, the effects of air recirculation due to the compensating subsiding motions in the cloud environment are underestimated. However, this error is much smaller than that originally introduced by the capping of the physical flows and can be diminished by applying a lower $f_{\text {maxfrac }}$. Additionally, applying the analytic expression accounts for the recirculation around the base of the updraft plume and reduces this error. The updated mixing ratios at the updraft base enhance the efficiency of the convective transport, but the induced deviations are of the same order as applying the analytic expression. The magnitudes of all induced differences depend on the lifetime of the evaluated atmospheric compound, which is related to the associated vertical distribution of the tracer and to the regions that are mainly affected by the applied modification. The intermediate time stepping proved most influential for lifetimes on the order of 1 day, while the other two modifications become more influential with shorter lifetimes.

Even though the analytic expression and updated plume base mixing ratios are not as important as intermediate time stepping and only result in root mean square deviations in the temporally averaged mixing ratios of less than $1 \%$ of the air-mass-weighted mixing ratios, these improvements come without extra computational cost. Furthermore, these metrics were determined for averaged mixing ratios over 2001, while local, instantaneous mixing ratios will likely differ more strongly. This will be of importance when comparing model data directly with time-dependent observations. For future numerical experiments we therefore recommend to enable all three modifications. Only when intermediate time stepping is disabled should the analytic expression not be applied to prevent a further underestimation of the convective transport. The optimal setting of $f_{\text {maxfrac }}$ depends on the selected submodels in EMAC. If more computationally expensive submodels are enabled, a lower $f_{\text {maxfrac }}$ will result in de- creased deviations without a noteworthy increase in computational time. In the evaluated numerical experiment a value of 0.5 was chosen.

As a future development of the convective transport, the current "leaky pipe" representation could be further investigated. In the current implementation, at every individual time step an independent realization of the convective updrafts and downdrafts is calculated. This could be updated to a plume that evolves in time, similar to the environmental air. Furthermore, it would be worthwhile to further quantify, and subsequently apply, the correct value for $f_{\mathrm{d}}$ for the various applied numerical grids. Finally, it has been shown that the convective cloud cover representation substantially affects the distribution of atmospheric compounds. Based on Cuijpers and Bechtold (1995), more representative estimates of this convective cloud cover have been proposed (e.g., Neggers et al., 2006). However, as discussed by Sikma and Ouwersloot (2015), these have to be further adapted. To accurately represent convective transport, it will be important to include these updated parameterizations.

\section{Code availability}

The Modular Earth Submodel System (MESSy) is being continuously further developed and applied by a consortium of institutions. The usage of MESSy and access to the source code is licensed to all affiliates of institutions that are members of the MESSy Consortium. Institutions can be a member of the MESSy Consortium by signing the Memorandum of Understanding. More information can be found on the MESSy Consortium website (http://www.messy-interface. org).

\section{The Supplement related to this article is available online at doi:10.5194/gmd-8-2435-2015-supplement.}

Acknowledgements. The authors thank Jordi Vilà-Guerau de Arellano and Martin Sikma for their feedback during this project. We further wish to acknowledge the use of the Ferret program (http://ferret.pmel.noaa.gov) for graphics in this paper.

The article processing charges for this open-access publication were covered by the Max Planck Society.

Edited by: V. Grewe

\section{References}

Arakawa, A.: The cumulus parameterization problem: past, present, and future, J. Climate, 17, 2493-2525, doi:10.1175/15200442(2004)017<2493:RATCPP>2.0.CO;2, 2004. 
Bechtold, P., Cuijpers, J. W. M., Mascart, P., and Trouilhet, P.: Modeling of trade wind cumuli with a low-order turbulence model: toward a unified description of $\mathrm{Cu}$ and $\mathrm{Sc}$ clouds in meteorological models, J. Atmos. Sci., 52, 455-463, doi:10.1175/15200469(1995)052<0455:MOTWCW>2.0.CO;2, 1995.

Cuijpers, J. W. and Bechtold, P.: A simple parameterization of cloud water related variables for use in boundary layer models, J. Atmos. Sci., 52, 2486-2490, doi:10.1175/15200469(1995)052<2486:ASPOCW>2.0.CO;2, 1995.

Jöckel, P., Sander, R., Kerkweg, A., Tost, H., and Lelieveld, J.: Technical Note: The Modular Earth Submodel System (MESSy) - a new approach towards Earth System Modeling, Atmos. Chem. Phys., 5, 433-444, doi:10.5194/acp-5-433-2005, 2005.

Jöckel, P., Tost, H., Pozzer, A., Brühl, C., Buchholz, J., Ganzeveld, L., Hoor, P., Kerkweg, A., Lawrence, M. G., Sander, R., Steil, B., Stiller, G., Tanarhte, M., Taraborrelli, D., van Aardenne, J., and Lelieveld, J.: The atmospheric chemistry general circulation model ECHAM5/MESSy1: consistent simulation of ozone from the surface to the mesosphere, Atmos. Chem. Phys., 6, 5067-5104, doi:10.5194/acp-6-5067-2006, 2006.

Jöckel, P., Kerkweg, A., Buchholz-Dietsch, J., Tost, H., Sander, R., and Pozzer, A.: Technical Note: Coupling of chemical processes with the Modular Earth Submodel System (MESSy) submodel TRACER, Atmos. Chem. Phys., 8, 1677-1687, doi:10.5194/acp8-1677-2008, 2008.

Jöckel, P., Kerkweg, A., Pozzer, A., Sander, R., Tost, H., Riede, H., Baumgaertner, A., Gromov, S., and Kern, B.: Development cycle 2 of the Modular Earth Submodel System (MESSy2), Geosci. Model Dev., 3, 717-752, doi:10.5194/gmd-3-717-2010, 2010.

Kerkweg, A., Sander, R., Tost, H., and Jöckel, P.: Technical note: Implementation of prescribed (OFFLEM), calculated (ONLEM), and pseudo-emissions (TNUDGE) of chemical species in the Modular Earth Submodel System (MESSy), Atmos. Chem. Phys., 6, 3603-3609, doi:10.5194/acp-6-3603-2006, 2006.

Kim, S.-W., Barth, M. C., and Trainer, M.: Influence of fair-weather cumulus clouds on isoprene chemistry, J. Geophys. Res., 117, D10302, doi:10.1029/2011JD017099, 2012.

Lawrence, M. G. and Rasch, P. J.: Tracer transport in deep convective updrafts: plume ensemble versus bulk formulations, J. Atmos. Sci., 62, 2880-2894, doi:10.1175/JAS3505.1, 2005.

Lelieveld, J. and Crutzen, P. J.: Role of deep cloud convection in the ozone budget of the troposphere, Science, 264, 1759-1761, doi:10.1126/science.264.5166.1759, 1994.
Neggers, R., Stevens, B., and Neelin, J. D.: A simple equilibrium model for shallow-cumulus-topped mixed layers, Theor. Comp. Fluid Dyn., 20, 305-322, doi:10.1007/s00162-006-0030-1, 2006.

Nordeng, T. E.: Extended Versions of the Convective Parametrization Scheme at ECMWF and Their Impact on the Mean and Transient Activity of the Model in the Tropics, Tech. Rep. 206, ECMWF, 1994.

Ouwersloot, H. G., Vilà-Guerau de Arellano, J., van Stratum, B. J. H., Krol, M. C., and Lelieveld, J.: Quantifying the transport of subcloud layer reactants by shallow cumulus clouds over the Amazon, J. Geophys. Res.-Atmos., 118, 13041-13059, doi:10.1002/2013JD020431, 2013.

Roeckner, E., Brokopf, R., Esch, M., Giorgetta, M., Hagemann, S., Kornblueh, L., Manzini, E., Schlese, U., and Schulzweida, U.: Sensitivity of simulated climate to horizontal and vertical resolution in the ECHAM5 atmosphere model, J. Climate, 19, 37713791, doi:10.1175/JCLI3824.1, 2006.

Siebesma, A. P. and Cuijpers, J. W.: Evaluation of parametric assumptions for shallow cumulus convection, J. Atmos. Sci., 52, 650-666, doi:10.1175/15200469(1995)052<0650:EOPAFS>2.0.CO;2, 1995.

Sikma, M. and Ouwersloot, H. G.: Parameterizations for convective transport in various cloud-topped boundary layers, Atmos. Chem. Phys. Discuss., 15, 10709-10738, doi:10.5194/acpd-1510709-2015, 2015.

Tiedtke, M.: A comprehensive mass flux scheme for cumulus parameterization in large-scale models, Mon. Weather Rev., 117, 1779-1800, doi:10.1175/15200493(1989)117<1779:ACMFSF>2.0.CO;2, 1989.

Tost, H., Jöckel, P., and Lelieveld, J.: Influence of different convection parameterisations in a GCM, Atmos. Chem. Phys., 6, 54755493, doi:10.5194/acp-6-5475-2006, 2006.

Tost, H., Lawrence, M. G., Brühl, C., Jöckel, P., The GABRIEL Team, and The SCOUT-O3DARWIN/ACTIVE Team: Uncertainties in atmospheric chemistry modelling due to convection parameterisations and subsequent scavenging, Atmos. Chem. Phys., 10, 1931-1951, doi:10.5194/acp-10-1931-2010, 2010.

Yanai, M., Esbensen, S., and Chu, J.-H.: Determination of bulk properties of tropical cloud clusters from large-scale heat and moisture budgets, J. Atmos. Sci., 30, 611-627, doi:10.1175/1520-0469(1973)030<0611:DOBPOT>2.0.CO;2, 1973. 